\title{
Generation of a Sequence of Correlated Phase Screens
}

\author{
Parisa Fatheddin*1,2 and Jonathan Gustafsson*3,4 \\ ${ }^{1}$ Department of Engineering Physics, Center for Directed Energy, \\ Wright-Patterson AFB, Dayton, OH 45433, USA. \\ ${ }^{2}$ Oak Ridge Institute for Science and Education, 1299 Bethel Valley \\ Road, Oak Ridge, TN 37380, USA. \\ ${ }^{3}$ Department of Mathematics and Statistics, Air Force Institute of \\ Technology, WPAFB, OH 45433, USA. \\ ${ }^{4}$ National Research Council, 500 Fifth Street NW, Washington, DC \\ 20001, USA.
}

\begin{abstract}
A novel technique is given and implemented to generate correlated phase screens that are used in the study of laser propagation through turbulent atmosphere. The method can generate random fields with nonzero expected values and is applied to simulate equally and arbitrary spaced phase screens. In both cases, it proves to be very computationally efficient.
\end{abstract}

Laser propagation through random media has seen extensive investigation. It has many important applications in the improvement of image resolution of telescopes, in industry in welding of metals and in medicine in laser surgery (see for example $[1,11,14,20]$ ). One can observe the significance in the precision of directing lasers through random media. To this end, lasers are modeled as electromagnetic waves whose refractive index become altered as they go through a medium as a cause of temperature and density fluctuations in the atmosphere. These alterations in turn change the phase of the waves, which have promoted the idea of dividing the medium

*Supported partially by Office of Naval Research's (ONR) Atmospheric Propagation Sciences for High Energy Lasers (APSHELS)

(C) 2017. This manuscript version is made available under the Elsevier user license http://www.elsevier.com/open-access/userlicense/1.0/ 
into phase screens indicating where the phase changes. The screens can be thought of as two or three dimensional layers or slabs. For more background on laser propagation through random media we recommend $[2,11,12,16]$.

To generate phase screens, many numerical simulation methods have been proposed. The classical approach is the Monte Carlo simulation which creates random arrays of phase values in a grid of sample points that have the same statistics as the turbulence-induced atmospheric phase. Here we will use Fourier filtering method, which is based on the Fast Fourier Transform (FFT) of the process with the requirement that the process be stationary. Similar to our result, X. Xiao and D. Voelz in [21] also applied the Fast Fourier Transform technique to simulate phase screens. They simulated uncorrelated phase screens to examine the intensity of laser light as it propagates. In addition, Dieker and Mandjes used this simulation approach in [4] to compute the discrete Fourier transform of one dimensional fractional Brownian motion $(\mathrm{fBm})$. Since $\mathrm{fBm}$ does not posses the stationary requirement, the stationary property of its increments given as white noise is used instead. For two and three dimensions, [22] gives this method for fBm using the turning band method, which he claims to be faster and more accurate than the Fourier filtering method; however, we find the Fourier filtering method more accessible and easier to implement. Here we provide details to Fourier filtering technique and apply it to generate two and three dimensional phase screens correlated by temperature. Our simulation technique has also been applied to a stationary Gaussian process in [6] and explained in [14]. We extend the results of [6] by not requiring the mean of the Gaussian random field to be zero. Chapter 12 of [15] also uses fast Fourier transform and provides detailed steps for the simulation of important types of spatial processes such as Gaussian and Markov random fields, point processes, spatial Wiener processes and Levy fields. Here we have a similar situation since the temperature at each point of the phase screen is assumed to be a stationary Gaussian random field. For more general simulation approaches for turbulence in random media see direct numerical simulation and large-eddy simulation in chapters 8 and 9 of [10]. In addition for other classical numerical simulation methods of phase screens see [7] and the references within.

Most previous work on the study of phase screens has been on uncorrelated phase screens. If the distance between phase screens is sufficiently small then the screens are guaranteed to be correlated. Intuitively it is clear that the correlation between phase screens increases as they are placed closer to each other. It is assumed that in the space between phase screens, the phase does not change. For examples of results based on uncorrelated phase 
screens see for example $[5,17,18]$.

Recently some sources have considered correlated phase screens. Naeh and Katzir in [8] proposed the Sparse Spectrum Harmonic Augmentation (SSHA) method, which simulates perfectly correlated phase screens in an isotropic three-dimensional media. The method compresses three-dimensional refractive index media to two-dimensional phase screens and for its implementation one needs the discrete frequency, amplitude, random phase and vector directions. Also [9] simulated correlated phase screens by calculating the auto-correlation (correlation between two points on the same screen) and cross-correlation (correlation between two points on two different phase screens) of change in phase. In contrast to our approach they do not take into account the Markov property of the phase screens. However, similar to our method they use the Fast Fourier transform to convert delta-correlated random numbers in the spatial domain to the spectral domain by using the relation between a phase screen with its spectrum by the Fourier transform pair,

$$
\begin{aligned}
\Phi(\mathbf{k}) & =\int p(\mathbf{r}) e^{-2 \pi i \mathbf{r} \cdot \mathbf{k}} d \mathbf{r} \\
p(\mathbf{r}) & =\int \Phi(\mathbf{k}) e^{2 \pi i \mathbf{k} \cdot \mathbf{r}} d \mathbf{k}
\end{aligned}
$$

where $\Phi(\mathbf{k})$ is the spectrum of the phase fluctuations and $p(\mathbf{r})$ is the phase screen at $\mathbf{r}=(x, y)$. Their simulated phase screens demonstrate that the larger pockets of air show more correlation, which is also what we have observed in our simulated phase screens. For the simulation of correlated phase screens see also [19]. Here we consider correlated phase screens based on temperature, where the distance between the screens is measured as a fraction of the integral length scale and is on the order of meters.

This article is organized as follows. Section one describes our new approach to Fourier filtering method. The application of this technique to generate correlated phase screens is given in section two. In section three, we analytically examine the effects of Markov property on estimating temperature at a fixed point on a phase screen along the path, knowing the temperature at this point on the screen before it. Then in section four, we provide an algorithm based on the new Fourier filtering method given in section one, that can be used to generate phase screens with Markov property. Section five provides a description on our numerical results along with our figures illustrating our simulations. We conclude with section six with a summary of our results. All of our findings can be applied to two and three dimensional phase screens. Without loss of generality, we focus on three 
dimensional screens and note that the results can be easily modified to two dimensional screens without requiring changes to the techniques.

\section{Fourier Filtering Method}

In this section we present a new approach to the Fourier filtering method. Suppose we have a real-valued stationary isotropic Gaussian random field $X(\mathbf{x})$ with $\mathbf{x}=(x, y, z)$ and domain being a three dimensional finite lattice, denoted as $\mathcal{D}$. In addition, suppose we are given that for all $\mathbf{x} \in \mathcal{D}$,

$$
\begin{aligned}
\mathbb{E}(X) & =0, \\
\mathbb{E}\left(X^{2}\right) & =\sigma^{2}<\infty,
\end{aligned}
$$

with autocorrelation,

$$
f(|\mathbf{r}|)=\mathbb{E}\left(X(\mathbf{x}) X\left(\mathbf{x}^{\prime}\right)\right)=\mathbb{E}(X(\mathbf{x}) X(\mathbf{x}-\mathbf{r}))
$$

where $\mathbf{x}$ and $\mathbf{x}^{\prime}$ are two position vectors and $|\mathbf{r}|=\left|\mathbf{x}-\mathbf{x}^{\prime}\right|$ is the distance between them. Autocorrelation is a function of $|\mathbf{r}|$, which we assume to be continuous and positive definite so that by Bochner's theorem (see Pg. 303 of [13]), its Fourier transform, $\hat{f}$, is real and positive.

By the zero mean assumption on $X$, we have, for a vector, $\mathbf{k}$ in the Fourier space,

$$
0=\mathcal{F}[\mathbb{E}(X)](\mathbf{k})=\mathbb{E}(\mathcal{F}[X](\mathbf{k}))=\mathbb{E}(\hat{X}(\mathbf{k})) .
$$

As an application of the convolution theorem for Fourier transforms we have,

$$
\begin{aligned}
\int_{\mathcal{D}} \hat{f}(|\mathbf{k}|) d \mathbf{x}= & \mathcal{F}\left(\mathbb{E}\left(\int_{\mathcal{D}} X(\mathbf{x}) X(\mathbf{x}-\mathbf{r}) d \mathbf{x}\right)\right) \\
& =\mathbb{E}\left(\mathcal{F}\left(\int_{\mathcal{D}} X(\mathbf{x}) X(\mathbf{x}-\mathbf{r}) d \mathbf{x}\right)\right)=\mathbb{E}(\hat{X}(\mathbf{k}) \cdot \overline{\hat{X}}(\mathbf{k}))
\end{aligned}
$$

so that,

$$
\hat{f}(|\mathbf{k}|)=\frac{\mathbb{E}(\hat{X}(\mathbf{k}) \cdot \overline{\hat{X}}(\mathbf{k}))}{\int_{\mathcal{D}} d \mathbf{x}}
$$

where we have used the Fubini's theorem repeatedly since by applying Cauchy-Schwartz inequality, we have $X(\mathbf{x}) X(\mathbf{x}-\mathbf{r}) \in L^{1}$. For the simplicity of notation, we take into account the finite assumption on $\mathcal{D}$ and let $\mu(\mathcal{D}):=\int_{\mathcal{D}} d \mathbf{x}<\infty$ be the Lebesgue measure of $\mathcal{D}$. 
Since $X(\mathbf{x})$ is a real-valued Gaussian random field, then its Fourier transform, $\hat{X}(\mathbf{k}):=U(\mathbf{k})+i V(\mathbf{k})$, is also Gaussian and by Cramer's theorem (see Theorem 6.19 of [3]), $U$ and $V$ are independent Gaussian random variables. Let $U(\mathbf{k}) \sim N\left(\mu_{1}(\mathbf{k}), \sigma_{1}^{2}(\mathbf{k})\right)$ and $V(\mathbf{k}) \sim N\left(\mu_{2}(\mathbf{k}), \sigma_{2}^{2}(\mathbf{k})\right)$. Since by assumption, $X$ has mean zero and variance, $\sigma^{2}<\infty$ then,

$$
\begin{array}{r}
\mathbb{E}(\hat{X}(\mathbf{k}))=0, \\
\mu_{1}(\mathbf{k})=\mu_{2}(\mathbf{k})=0 .
\end{array}
$$

Let $\tilde{\sigma}^{2}(\mathbf{k})$ denote the variance of $\hat{X}(\mathbf{k})$ then, we have, using the independence property of $U(\mathbf{k})$ and $V(\mathbf{k})$,

$$
\begin{aligned}
\mathbb{E}(\hat{X}(\mathbf{k}) \cdot \overline{\hat{X}}(\mathbf{k}))=\mathbb{E}\left(U^{2}(\mathbf{k})+V^{2}(\mathbf{k})\right) & \\
\mathbb{E}(U(\mathbf{k}))^{2}+\mathbb{E}(V(\mathbf{k}))^{2} & +\sigma_{1}^{2}(\mathbf{k})+\sigma_{2}^{2}(\mathbf{k})= \\
& \mu_{1}^{2}(\mathbf{k})+\mu_{2}^{2}(\mathbf{k})+\tilde{\sigma}^{2}(\mathbf{k})=\tilde{\sigma}^{2}(\mathbf{k})
\end{aligned}
$$

so by equation (5) we have,

$$
\mu(\mathcal{D}) \hat{f}(|\mathbf{k}|)=\mathbb{E}(\hat{X}(\mathbf{k}) \cdot \overline{\hat{X}}(\mathbf{k}))=\tilde{\sigma}^{2}(\mathbf{k})
$$

giving, $\hat{X}(\mathbf{k}) \sim N(0, \mu((D)) \hat{f}(|\mathbf{k}|))+i N(0, \mu(\mathcal{D}) \hat{f}(|\mathbf{k}|))$ and $f$ being positive definite makes $\hat{f}$ real and positive assuring that the variance is real and positive.

Now assume we are given a real-valued Gaussian random field, $X(\mathbf{x})$ with the same properties as in the previous case with the exception that $\mathbb{E}(X(\mathbf{x}))=g(\mathbf{x})$, where $g$ is a given real function. With the same equalities as in (3), we obtain,

$$
\mathbb{E}(\hat{X}(\mathbf{k}))=\hat{g}(\mathbf{k}) .
$$

Using the Gaussian distribution for the Fourier transform of $X$ as in the previous case, we have $\hat{g}(\mathbf{k})=\mu_{1}(\mathbf{k})+i \mu_{2}(\mathbf{k})$ and by (5) and similar to (7),

$$
\begin{aligned}
& \mu(\mathcal{D}) \hat{f}(|\mathbf{k}|)=\mathbb{E}(\hat{X}(\mathbf{k}) \cdot \overline{\hat{X}}(\mathbf{k}))= \\
& \mu_{1}^{2}(\mathbf{k})+\mu_{2}^{2}(\mathbf{k})+\tilde{\sigma}^{2}(\mathbf{k})=|\hat{g}(\mathbf{k})|^{2}+\tilde{\sigma}^{2}(\mathbf{k})
\end{aligned}
$$

so that

$$
\begin{aligned}
\hat{X}(\mathbf{k}) & \sim N\left(\operatorname{Re}(\hat{g}(\mathbf{k})), \mu(\mathcal{D}) \hat{f}(|\mathbf{k}|)-|\hat{g}(\mathbf{k})|^{2}\right) \\
& +i N\left(\operatorname{Im}(\hat{g}(\mathbf{k})), \mu(\mathcal{D}) \hat{f}(|\mathbf{k}|)-|\hat{g}(\mathbf{k})|^{2}\right)
\end{aligned}
$$


To confirm the positivity of $\mu(\mathcal{D}) \hat{f}(|\mathbf{k}|)-|\hat{g}(\mathbf{k})|^{2}$, observe that,

$$
\begin{array}{r}
|\hat{g}(\mathbf{k})|^{2}=|\mathbb{E}(\hat{X}(\mathbf{k}))|^{2}=|\mathbb{E}(U(\mathbf{k}))+i \mathbb{E}(V(\mathbf{k}))|^{2}=\mathbb{E}(U(\mathbf{k}))^{2}+\mathbb{E}(V(\mathbf{k}))^{2} \\
\leq \mathbb{E}\left(U^{2}(\mathbf{k})\right)+\mathbb{E}\left(V^{2}(\mathbf{k})\right)=\mathbb{E}(\hat{X}(\mathbf{k}) \cdot \hat{\bar{X}}(\mathbf{k}))=\mu(\mathcal{D}) \hat{f}(|\mathbf{k}|)
\end{array}
$$

thus, limiting the choice of $g$.

\section{Generation of Temperature Values on a Fixed Phase Screen}

We apply the Fourier filtering method presented in the previous section to generate phase screens in a three dimensional temperature field, denoted as $\mathcal{D}$. We assume the phase screens to be correlated based on temperature and the distance between them to be the same. Let $\mathbf{x}^{\prime}=\left(x^{\prime}, y^{\prime}\right)$ be a fixed point on phase screen at $z$ and $\mathbf{x}=(x, y)$ be any other point on this screen. Furthermore, denote the temperature at position $\mathbf{x}$ in the screen at point $z$ as $T(\mathbf{x}, z)$. According to section one, suppose we are given,

$$
\begin{aligned}
\mathbb{E}\left(T\left(\mathbf{x}^{\prime}, z\right)\right) & =g\left(\mathbf{x}^{\prime}, z\right) \\
\mathbb{E}\left(T\left(\mathbf{x}^{\prime}, z\right)^{2}\right)-g\left(\mathbf{x}^{\prime}, z\right)^{2} & =\sigma^{2}<\infty
\end{aligned}
$$

and that the autocorrelation is provided as,

$$
\mathbb{E}\left(T(\mathbf{x}, z) T\left(\mathbf{x}^{\prime}, z^{\prime}\right)\right)=f(|\mathbf{r}|)=f\left(\sqrt{\left(x-x^{\prime}\right)^{2}+\left(y-y^{\prime}\right)^{2}+\left(z-z^{\prime}\right)^{2}}\right) .
$$

We use the following algorithm to generate a random temperature field.

1. Take the Fourier transform of the autocorrelation:

$$
\mathcal{F}\left[\mathbb{E}\left(T(\mathbf{x}, z) T\left(\mathbf{x}^{\prime}, z^{\prime}\right)\right)\right]=\mathcal{F}[f(|\mathbf{r}|)]=\hat{f}(|\mathbf{k}|)
$$

2. Let

$$
\hat{T}(\mathbf{k})=U(\mathbf{k})+i V(\mathbf{k})
$$

where

$$
\begin{aligned}
& U \sim N\left(\operatorname{Re}(\hat{g}(\mathbf{k})), \max \left\{\mu(\mathcal{D}) \hat{f}(|\mathbf{k}|)-|\hat{g}(\mathbf{k})|^{2}, 0\right\}\right), \\
& V \sim N\left(\operatorname{Im}(\hat{g}(\mathbf{k})), \max \left\{\mu(\mathcal{D}) \hat{f}(|\mathbf{k}|)-|\hat{g}(\mathbf{k})|^{2}, 0\right\}\right) .
\end{aligned}
$$


3. A random temperature field with the given autocorrelation can be generated by,

$$
T(\mathbf{x}, z)=\mathcal{F}^{-1}[\hat{T}(\mathbf{k})]
$$

Hence, knowing the autocorrelation between two points and the average value, we can generate values for the temperature at any point in $\mathcal{D}$.

\section{Correlated Phase Screens with Markov Prop- erty}

In this section we investigate the correlation of phase screens based on temperature, by examining it analytically. The screens can be thought of as sheets in the two dimensional case and rectangular boxes having volume in the three dimensional case. We assume the screens to have equal size arranged with different distance between them in the $z$ direction. Based on experimental data, the temperature at each point on a phase screen is Gaussian with possibly nonzero mean. We assume that the screens have the Markov property so that the temperature at a screen only depends on the known temperature of the latest phase screen before it.

Let $\mathbf{x}^{\prime}$ be a fixed point on the phase screen at $z_{i}$. Given temperature $T\left(\mathbf{x}^{\prime}, z_{i}\right)$, we estimate the temperature at the fixed point, $\mathbf{x}^{\prime}$, on a screen at $z_{i+1}$, which is further along the $z$ direction by distance $\left|z_{i+1}-z_{i}\right|$. To match the setting of the Fourier filtering method, introduced in the previous section, we assume that we are given,

$$
\mathbb{E}\left(T\left(\mathbf{x}^{\prime}, z_{i}\right) T\left(\mathbf{x}^{\prime}, z_{i+1}\right)\right)=f\left(\left|z_{i+1}-z_{i}\right|\right),
$$

where, as required by the method, $f$ is a continuous, positive definite real function. We determine,

$$
\begin{array}{r}
f\left(\left|z_{i+1}-z_{i}\right|\right)=\mathbb{E}\left(T\left(\mathbf{x}^{\prime}, z_{i}\right) T\left(\mathbf{x}^{\prime}, z_{i+1}\right)\right) \\
=\mathbb{E}\left(\mathbb{E}\left(T\left(\mathbf{x}^{\prime}, z_{i}\right) T\left(\mathbf{x}^{\prime}, z_{i+1}\right) \mid T\left(\mathbf{x}^{\prime}, z_{0}\right)=t_{z_{0}}, \ldots, T\left(\mathbf{x}^{\prime}, z_{i}\right)=t_{z_{i}}\right)\right) \\
=\mathbb{E}\left(T\left(\mathbf{x}^{\prime}, z_{i}\right) \mathbb{E}\left(T\left(\mathbf{x}^{\prime}, z_{i+1}\right) \mid T\left(\mathbf{x}^{\prime}, z_{0}\right)=t_{z_{0}}, \ldots, T\left(\mathbf{x}^{\prime}, z_{i}\right)=t_{z_{i}}\right)\right) \\
\quad=\mathbb{E}\left(T\left(\mathbf{x}^{\prime}, z_{i}\right) \mathbb{E}\left(T\left(\mathbf{x}^{\prime}, z_{i+1}\right) \mid T\left(\mathbf{x}^{\prime}, z_{i}\right)=t_{z_{i}}\right)\right)
\end{array}
$$

where $t_{z_{i}}$ is the temperature at the point $\mathbf{x}^{\prime}$ on the screen at $z_{i}$ and the Markov property has been applied. Since $\mathbb{E}\left(T\left(\mathbf{x}^{\prime}, z_{i+1}\right) \mid T\left(\mathbf{x}^{\prime}, z_{i}\right)=t_{z_{i}}\right)=$ $h\left(T\left(\mathbf{x}^{\prime}, z_{i}\right)\right)$ for some real function, $h$, we obtain,

$$
f\left(\left|z_{i+1}-z_{i}\right|\right)=\mathbb{E}\left(T\left(\mathbf{x}^{\prime}, z_{i}\right) T\left(\mathbf{x}^{\prime}, z_{i+1}\right)\right)=\mathbb{E}\left(T\left(\mathbf{x}^{\prime}, z_{i}\right) h\left(T\left(\mathbf{x}^{\prime}, z_{i}\right)\right)\right)
$$


Now based on the given information, the only candidate for $h\left(T\left(\mathbf{x}^{\prime}, z_{i}\right)\right)$, that would not require more assumptions on the temperature, is $h\left(T\left(\mathbf{x}^{\prime}, z_{i}\right)\right)=$ $K T\left(\mathbf{x}^{\prime}, z_{i}\right)$ where $K$ is a positive constant. Using equation (20), $K=$ $f\left(\left|z_{i+1}-z_{i}\right|\right) / \mathbb{E}\left(T\left(\mathbf{x}^{\prime}, z_{i}\right)^{2}\right)$, thus, we obtain,

$$
\mathbb{E}\left(T\left(\mathbf{x}^{\prime}, z_{i+1}\right) \mid T\left(\mathbf{x}^{\prime}, z_{i}\right)=t_{z_{i}}\right)=h\left(T\left(\mathbf{x}^{\prime}, z_{i}\right)\right)=\frac{f\left(\left|z_{i+1}-z_{i}\right|\right)}{f(0)} T\left(\mathbf{x}^{\prime}, z_{i}\right)
$$

where $f(0)=\mathbb{E}\left(T\left(\mathbf{x}^{\prime}, z_{i}\right)^{2}\right)>0$ and by the positive definite property of $f$, $\left|f\left(\left|z_{i+1}-z_{i}\right|\right)\right|<f(0)$.

If the distance between each phase screen is constant we can use the Markov property to find the correlation between all screens. We let this equal distance be denoted by an integer $\alpha>1$. To determine the correlation between phase screens at $z_{i}$ and $z_{i+\alpha}$ we have,

$$
\begin{aligned}
& \mathbb{E}\left(T\left(\mathbf{x}^{\prime}, z_{i}\right) T\left(\mathbf{x}^{\prime}, z_{i+\alpha}\right)\right) \\
&=\mathbb{E}\left(T\left(\mathbf{x}^{\prime}, z_{i}\right) \mathbb{E}\left(T\left(\mathbf{x}^{\prime}, z_{i+\alpha}\right) \mid T\left(\mathbf{x}^{\prime}, z_{i+\alpha-1}\right)=t_{z_{i+\alpha-1}}\right)\right) \\
&=\mathbb{E}\left(T\left(\mathbf{x}^{\prime}, z_{i}\right) h\left(T\left(\mathbf{x}^{\prime}, z_{i+\alpha-1}\right)\right)\right) .
\end{aligned}
$$

Similar to above we pick $h\left(T\left(\mathbf{x}^{\prime}, z_{i+\alpha-1}\right)\right)=K T\left(\mathbf{x}^{\prime}, z_{i+\alpha-1}\right)$ arriving at,

$$
\mathbb{E}\left(T\left(\mathbf{x}^{\prime}, z_{i}\right) T\left(\mathbf{x}^{\prime}, z_{i+\alpha}\right)\right)=K \mathbb{E}\left(T\left(\mathbf{x}^{\prime}, z_{i}\right) T\left(\mathbf{x}^{\prime}, z_{i+\alpha-1}\right)\right) .
$$

Therefore, repeating this process $\alpha$ times yields,

$$
\mathbb{E}\left(T\left(\mathbf{x}^{\prime}, z_{i}\right) T\left(\mathbf{x}^{\prime}, z_{i+\alpha}\right)\right)=K^{\alpha} \mathbb{E}\left(T\left(\mathbf{x}^{\prime}, z_{i}\right)^{2}\right)
$$

which confirms the exponential behavior we have observed in figure 1 .

\section{Generation of Correlated Phase Screens with Markov Property}

Here we generate arbitrary spaced phase screens having Markov property and apply our results from section 3. Suppose phase screens are not necessarily equally spaced. Suppose we have (12a) and (12b) and in addition, assume we are given the autocorrelation function and the correlation between phase screens at $z_{i}$ and $z_{i-1}$ as,

$$
\begin{aligned}
\mathbb{E}\left(T\left(\mathbf{x}, z_{i}\right) T\left(\mathbf{x}^{\prime}, z_{i}\right)\right) & =f\left(\left|\mathbf{x}-\mathbf{x}^{\prime}\right|\right), \\
h\left(T\left(\mathbf{x}^{\prime}, z_{i}\right)\right) & =\mathbb{E}\left(T\left(\mathbf{x}^{\prime}, z_{i}\right) \mid T\left(\mathbf{x}^{\prime}, z_{i-1}\right)=t_{z_{i-1}}\right) \\
& =\frac{f\left(\left|z_{i}-z_{i-1}\right|\right)}{f(0)} T\left(\mathbf{x}^{\prime}, z_{i-1}\right) .
\end{aligned}
$$

To generate correlated phase screens in this setting we follow the steps below. 


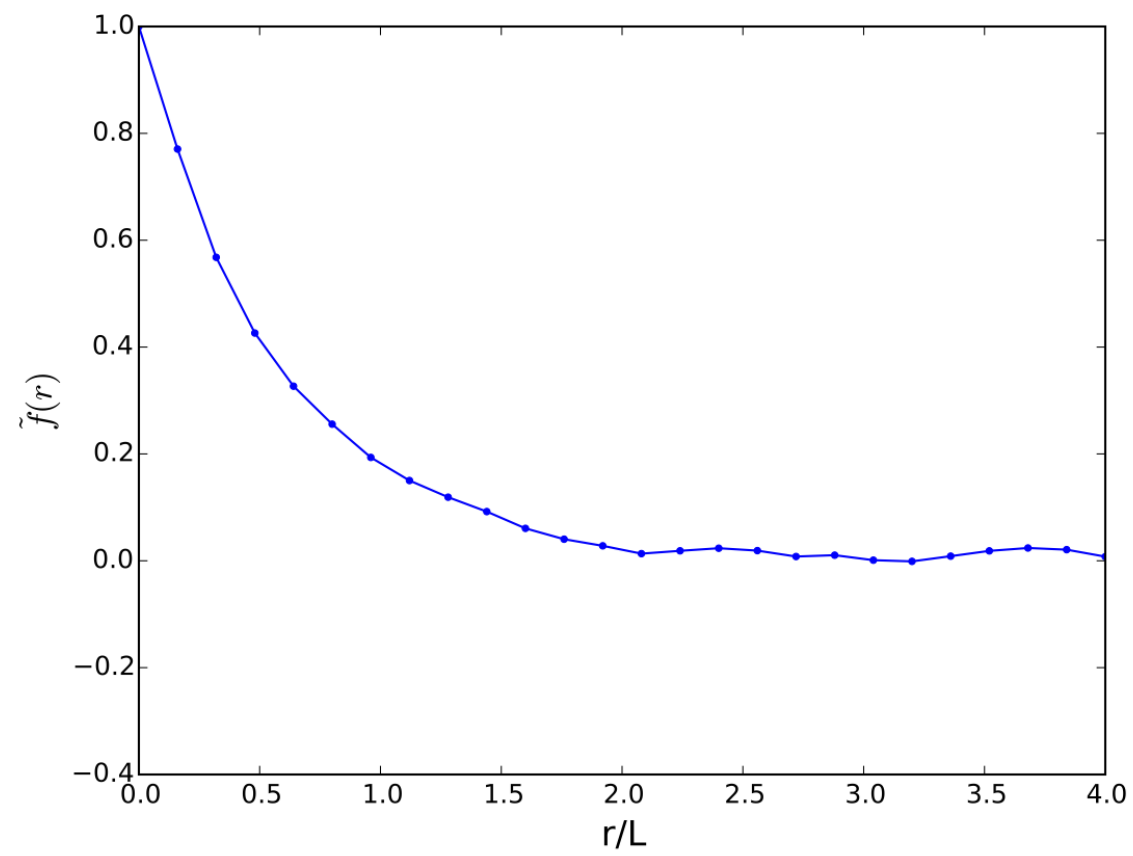

Figure 1: Numerically calculated correlation between the phase screen as a function of integral length scale. 
1. Take the Fourier transform of the autocorrelation function:

$$
\mathcal{F}\left[\mathbb{E}\left(T\left(\mathbf{x}, z_{i}\right) T\left(\mathbf{x}^{\prime}, z_{i}\right)\right)\right]=\mathcal{F}[f(|\mathbf{r}|)]=\hat{f}(|\mathbf{k}|)
$$

2. Make the phase screen at $z_{i}$ be dependent on the previous phase screen, $z_{i}$ :

$$
\hat{h}\left(\mathbf{k}, z_{i}\right)= \begin{cases}g\left(\mathbf{k}, z_{0}\right), & \text { if } i=0, \\ g\left(\mathbf{k}, z_{i}\right)+\frac{f\left(\left|z_{i}-z_{i-1}\right|\right)}{f(0)} \hat{T}\left(\mathbf{x}^{\prime}, z_{i-1}\right), & \text { else. }\end{cases}
$$

Note that $i=0$ indicates the first phase screen.

3. Let,

$$
\hat{T}\left(\mathbf{k}, z_{i}\right)=U(\mathbf{k})+i V(\mathbf{k})
$$

where,

$$
\begin{aligned}
& U \sim N\left(\operatorname{Re}\left(\hat{h}\left(\mathbf{k}, z_{i}\right)\right), \max \left\{\mu(\mathcal{D}) \hat{f}(|\mathbf{k}|)-\left|\hat{h}\left(\mathbf{k}, z_{i}\right)\right|^{2}, 0\right\}\right), \\
& V \sim N\left(\operatorname{Im}\left(\hat{h}\left(\mathbf{k}, z_{i}\right)\right), \max \left\{\mu(\mathcal{D}) \hat{f}(|\mathbf{k}|)-\left|\hat{h}\left(\mathbf{k}, z_{i}\right)\right|^{2}, 0\right\}\right) .
\end{aligned}
$$

4. The phase screen at position $z_{i}$ is obtained by,

$$
\mathcal{F}^{-1}\left(\hat{T}\left(\mathbf{k}, z_{i}\right)\right)=T\left(\mathbf{x}, z_{i}\right)
$$

5. Repeat steps 2 to 4 until all phase screens have been generated.

\section{$5 \quad$ Numerical Results}

We now provide phase screens that we have simulated using the method described. Figures 3 and 2 illustrate phase screens generated by algorithms presented by sections two and four, respectively, using the three dimensional spectrum given by [10, p. 232],

$$
\begin{aligned}
E(\kappa)=\oint_{\kappa}\left|\hat{f}\left(k_{1}, k_{2}, k_{3}\right)\right|^{2} \kappa^{2} \sin \phi d \phi d \theta & \\
& =\left(\frac{\kappa L}{\sqrt{(\kappa L)^{2}+c_{L}}}\right)^{5 / 3+p_{0}} \kappa^{-5 / 3} e^{-\beta \kappa l_{K}},
\end{aligned}
$$

where $\kappa=\sqrt{k_{1}^{2}+k_{2}^{2}+k_{3}^{2}}$. The above parameters determine the temperature spectrum and are set as, 
- $L$ : integral length scale, here $50 \mathrm{~m}$,

- $l_{K}$ : Kolmogorov micro-scale, here $1 \mathrm{~mm}$,

- $c_{L}$ : a positive constant, depending on integral length scale, here 6.78,

- $p_{0}$ : a positive constant, determining the power of the three dimensional energy function spectrum and as $\kappa \rightarrow 0, p_{0}=4$,

- $\beta=5.2$

- $c_{\eta}=0$

No wrapping mitigation techniques were utilized. In both figures 2 and 3 , the phase screens on the top left represent the screen at point $z_{i}$ and the one on the top right is the screen at $z_{i+1}$. The difference between the screens is in bottom left. Correlation between the slices is clear and an estimate for this correlation is given by,

$$
\tilde{f}(r)=\frac{1}{N_{1} N_{2} N_{3}} \sum_{i=0}^{N_{1}} \sum_{j=0}^{N_{2}} \sum_{k=0}^{N_{3}} T\left(x_{i}, y_{j}, z_{k}\right) T\left(x_{i}, y_{z}, z_{k}+r\right),
$$

where $N_{1}, N_{2}$ and $N_{3}$ represent the number of grid points in $x, y$ and $z$, respectively. The estimated correlation between slices are plotted against the integral length scale and are shown in the bottom right of figures 2 and 3 .

\section{Results}

In this article we described a technique for generating temperature fields with a known correlation function. The traditional method is to create a correlation matrix and factorize the matrix using the Cholesky factorization. The algorithm described here only uses the Fast Fourier Transform and is computationally faster and requires less memory. We also presented a method for generating correlated phase screens based on the Markov property. The new method can also generate random fields with non-zero and spatial varying mean.

\section{Acknowledgements}

We would like to thank Dr. Steven Fiorino for his support as the director of the Center for Directed Energy (CDE) at the Air Force Institute of 

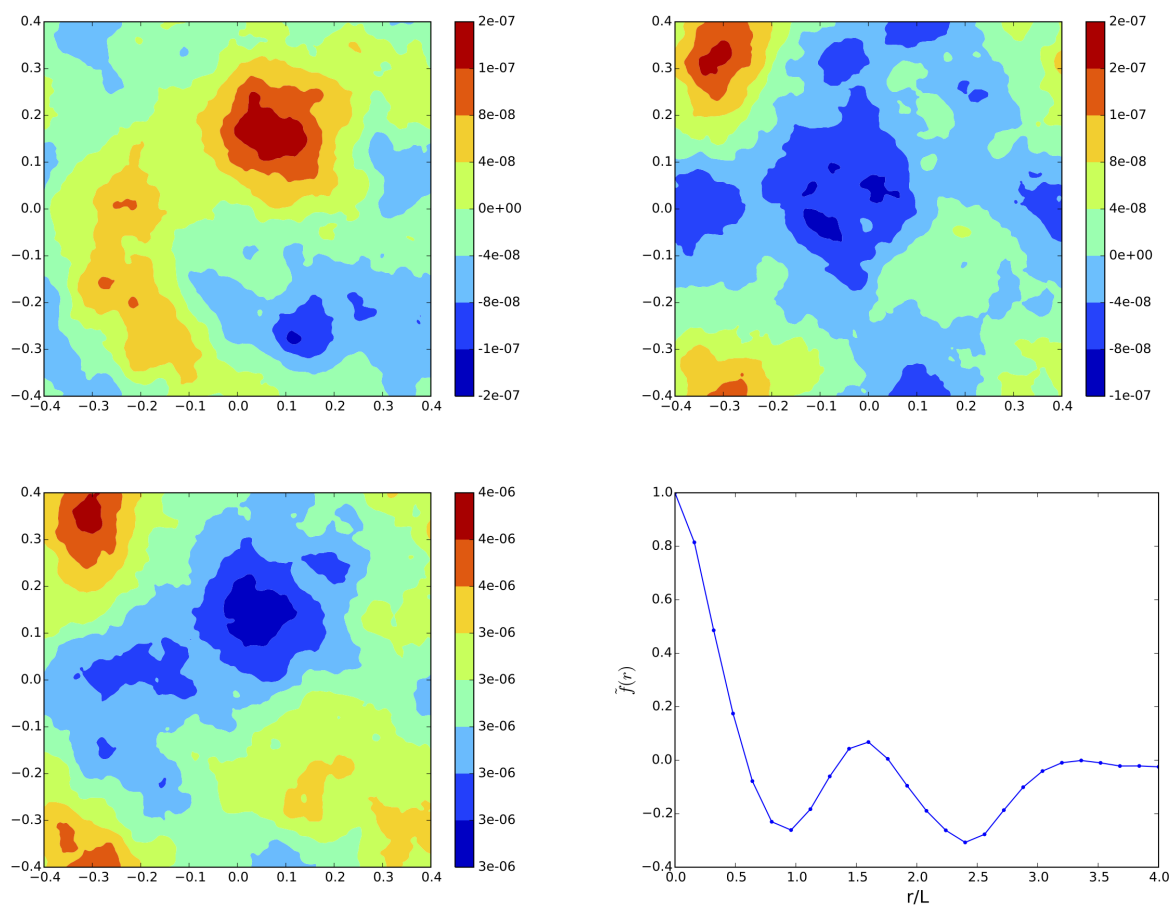

Figure 2: Slices of temperature field generated by the algorithm described in section 2. No wrapping mitigation techniques was utilized. Top left is the first slice. Top right is the second slice. Bottom left is the difference between the two slices. Bottom right is the numerically calculated correlation between the temperature slices as a function of integral length scale. 

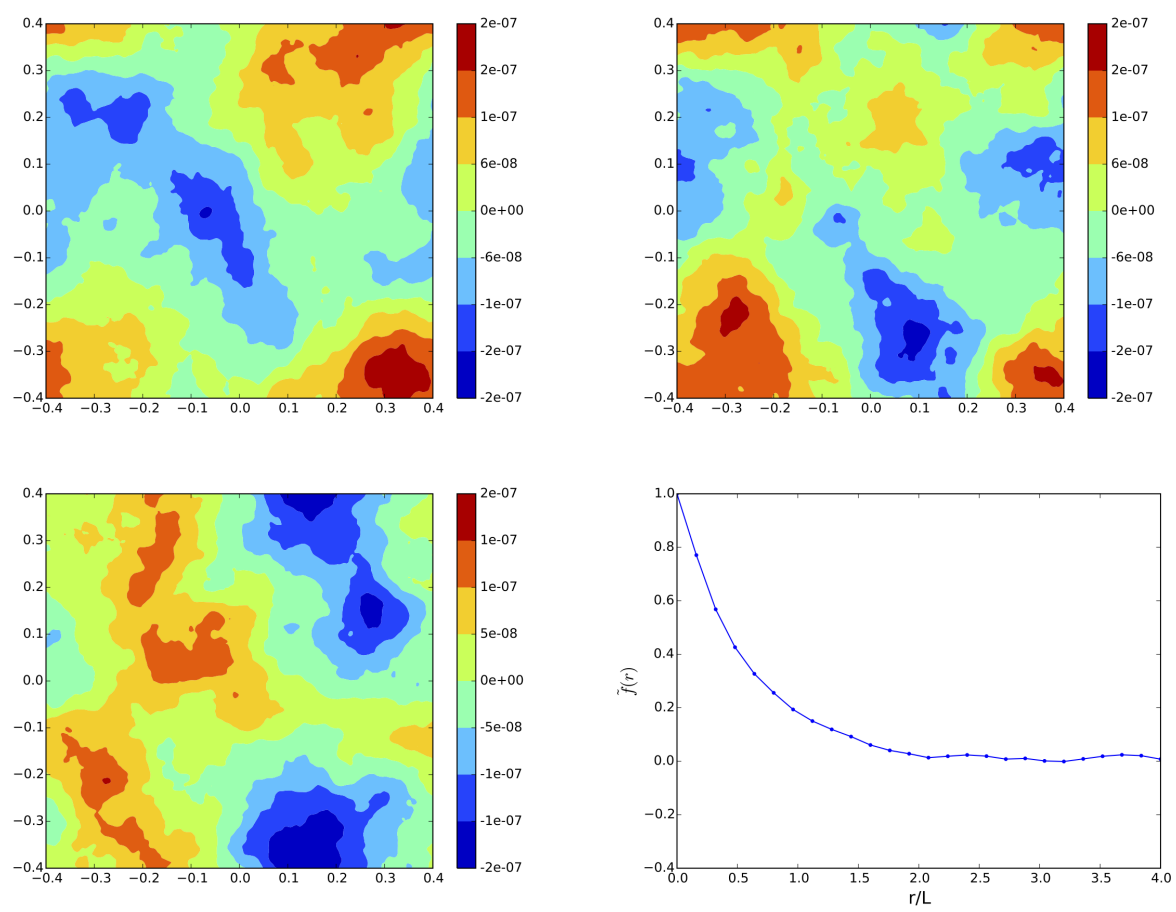

Figure 3: Sequence of phase screens described in section 4. Phase screens using the spectrum defined in equation (31). No wrapping mitigation techniques was utilized. Top left is the first phase screen. Top right is the second phase screen. Bottom left is the difference between the phase screens. Bottom right is the numerically calculated correlation between the phase screens as a function of integral length scale. 
Technology (AFIT). Dr. Fiorino and team members of CDE, such as Dr. Santasri Basu and Dr. Jack McCrae offered helpful conversations and insight into the physics of laser related problems and the one described here. CDE provided us valuable resources and dynamic research atmosphere to conduct our research. We also like to thank the editor and an anonymous referee for their comments and insights. The first author is thankful to Oak Ridge Institute for Science and Education (ORISE) and the second author is grateful to National Research Council (NRC) for making this research possible by serving as an interagency between the U.S. Department of Energy and AFIT and effectively taking care of its administrative tasks.

\section{References}

[1] A. Zardecki and C. Delisle (1977). Higher order statistics of light scattered by a random phase screen. Opt. Acta. vol. 24, no. 3, 241-259.

[2] L. Andrews and R. Phillips (2005). Laser Beam Propagation Through Random Media. The International Society for Optical Engineering (SPIE) Press.

[3] H. Cramer (1970). Random Variables and Probability Distributions. Cambridge At the University Press.

[4] A. Dieker and M. Mandjes (2003). On spectral simulation of fractional Brownian motion. Probab. Engrg. Inform. Sci. vol. 17, no. 3, 417-434.

[5] A. Dipankar and P. Sagaut (2009). A new phase-screen method for electromagnetic wave propagation in turbulent flows using large-eddy simulation. J. Comput. Phys. vol. 228, no. 20, 7729-7741.

[6] C. Dietrich and G. Newsam (1997). Fast and exact simulation of stationary Gaussian processes through circulant embedding of the covariance matrix. SIAM J. Sci. Comput. vol. 18, no. 4, 1088-1107.

[7] R. Lane, A. Glindemann and J. Dainty (1992). Simulation of a Kolmogorov phase screen. Waves in Random Media. vol. 2, no. 3, 209-224.

[8] I. Naeh and A. Katzir (2014). Perfectly correlated phase screen realization using sparse spectrum harmonic augmentation. Appl. Opt. vol. 53, no. $27,6168-6174$. 
[9] P. Paramonov, A. Vorontsov, and V. Kunitsyn (2015). A threedimensional refractive index model for simulation of optical wave propagation in atmospheric turbulence. Waves in Random and Complex Media vol. 25, no. 4, 556-575.

[10] S. Pope (2000). Turbulent Flows. Cambridge University Press.

[11] M. Roggemann and B. Welsh (1996). Imaging Through Turbulence. CRC Press: Laser and Optical Science and Technology Series.

[12] F. Roux (2014). The Lindblad equation for the decay of entanglement due to atmospheric scintillation. J. Phys. A. vol. 47, no. 19, 15pp.

[13] W. Rudin (1991). Functional Analysis: Second Edition. International Series in Pure and Applied Mathematics.

[14] J. Schmidt (2010). Numerical Simulation of Optical Wave Propagation with Examples in Matlab. SPIE Press.

[15] V. Schmidt (2014). Stochastic Geometry, Spatial Statistics and Random Fields. Lecture Notes in Mathematics, vol. 2120.

[16] J. Strohbehn (1978). Laser Beam Propagation in the Atmosphere. Topics in Applied Physics. Vol. 25, Springer, New York.

[17] B. Uscinski (1985). Analytical solution of the fourth-moment equation and interpretation as a set of phase screens. J. Opt. Soc. Am. A vol. 2, no. $12,2077-2091$.

[18] A. Vorontsov, P. Paramonov, M. Valley, and M. Vorontsov (2008). Generation of infinitely long phase screens for modeling of optical wave propagation in atmospheric turbulence. Waves in Random and Complex Media. vol. 18, no. 1, 91-108.

[19] B. Welsh (1997). Fourier-series-based atmospheric phase screen generator for simulating anisoplanatic geometries and temporal evolution.Proc. SPIE. vol. 3125.

[20] A. Wild, R. Hobbs and L. Frenje (2000). Modelling complex media: an introduction to the phase-screen method. Phys. Earth Planet. Inter. vol. 120, no. 3, 219-225.

[21] X. Xiao and D. Voelz (2006). Wave optics simulation approach for partial spatially coherent beams. Optics Express. vol. 14, no. 16, 6986-6992. 
[22] Z. Yin (1996). New methods for simulation of fractional Brownian motion. J. of Comput. Phys. vol. 127, no. 1, 66-72. 